\title{
Mixed-etiology leg ulcers in a patient on long-term glucocorticoid therapy
}

\author{
Piotr Sławiński, Marcin Radkowski, Andrzej Lewandowicz, Tomasz Targowski \\ Department of Geriatrics, National Institute of Geriatrics, Rheumatology and Rehabilitation, Warsaw, Poland
}

\begin{abstract}
Chronic leg ulceration is a frequent condition in elderly patients. Chronic wounds that are nonresponsive to 3-month therapy affect approximately 6.5 million people in the United States with a prevalence of $1 \%$ and costs estimated at 25 billion dollars per year. Although the main causes are venous insufficiency, lower extremity arterial disease and diabetes, in many cases the etiology is multi-factorial. Approximately $20-23 \%$ of non-healing wounds that are refractory to vascular intervention have other etiologies including vasculitis, rheumatoid arthritis and Sjögren syndrome. Adverse drug interactions are the least commonly considered, especially those which involve disease-modifying anti-rheumatic drugs.

The authors present a report on a female patient with reported Sjögren syndrome, multiple morbidities and non-healing lower limb ulceration that developed during treatment with methotrexate, and no significant improvement after discontinuation of the drug and after vascular surgery. Microvascular deterioration caused by beta-blockers was considered decisive. Calcium-blocker replacement brought complete healing in the follow-up.
\end{abstract}

Key words: microcirculation disorders, leg ulcers, chronic ulcers, beta-blockers.

\section{Introduction}

Chronic ulceration of lower limbs is a frequent condition with a prevalence of $3-5 \%$ in the population over 65 [1] and about $8 \%$ of elderly patients aged 85 and over. Around $50-70 \%$ of treated ulcers heal within 4-6 months, and $8 \%$ heal over the next 5 years [2]. Chronic wounds that are unresponsive to 3 months of appropriate wound care affect approximately 6.5 million people in the United States of America (US) with a prevalence of $1 \%$ and costs estimated at 25 billion dollars per year [3]. Although the main causes are venous insufficiency, lower extremity arterial disease and diabetes, in many cases the etiology is multi-factorial. In a study in the London population of patients with leg ulcers, venous disease was diagnosed simultaneously with arterial disease, diabetes, lymphedema and rheumatoid arthritis; in $35 \%$ of cases more than one cause of leg ulcers occurred [4].
Legs ulcers are a frequent problem in patients with rheumatoid arthritis (RA) and in patients with systemic sclerosis. Approximately $10 \%$ of patients with RA experienced leg ulceration. Larger studies have found that at least half of the ulcers in these patients are of multifactorial etiology. Approximately $10 \%$ of patients with RA are affected by leg ulcers while as many as $10 \%$ of leg ulcers can be associated with RA [5]. Approximately $20-23 \%$ of non-healing wounds that are refractory to vascular intervention have other etiology including vasculitis, RA, scleroderma, systemic lupus erythematosus, psoriasis, pyoderma gangrenosum [6], and Sjögren syndrome [7]. Thus, for proper treatment of patients with leg ulcers, it is important to be aware of the differential diagnosis of leg ulceration.

This article presents a patient with diagnosis of Sjögren's syndrome, multiple other morbidities and non-healing lower limb ulceration of mixed etiology and 
highlights the importance of drug selection to optimize microcirculation in anti-ulcerative treatment.

\section{Case report}

An 82-year-old woman with a long history of sicca syndrome diagnosis, metabolic syndrome comprising obesity, arterial hypertension, and hyperlipidemia, as well as Hashimoto thyroiditis with thyroid insufficiency, congestive heart failure, chronic kidney disease, bilateral cataract and lower limb ulcers, was admitted to the Department of Geriatrics in July 2016 due to symptoms of acute circulatory failure with pitting edema of lower limbs.

In the last 10-year period, the patient had undergone long-term glucocorticosteroid treatment for Sjögren syndrome. There was no available medical documentation for this period. In 2014, the patient had been hospitalized in the rheumatology department because of acute exacerbation of arthralgia and myalgia shortly after a sudden glucocorticosteroids withdrawal. There were symmetrical hand arthralgias without other signs of inflammation but with an elevated erythrocyte sedimentation rate and $\mathrm{C}$-reactive protein concentration, with negative rheumatoid factor (RF), negative anti-cyclic citrullinated peptide antibodies (ACPA) and antinuclear antibodies (ANA) titer 1:80 (Table I). Comparative hand (Fig. 1) and feet radiograms and spine radiograms showed mainly osteoarthritic changes with multiple geodes. Radiograms also revealed calcifications in arterial walls. Schirmer's test showed decrease of tear secretion ( $3 \mathrm{~mm} / 5 \mathrm{~min}$ in both eyes); there were signs and symptoms of conjunctivitis sicca.

There was no objective confirmation of mouth dryness. The described patient did not agree to a biopsy of the minor salivary glands. The patient did not meet ACR/EULAR 2010 [8] criteria for diagnosis of RA. The patient was diagnosed with unclassified arthritis and suc- cessfully treated with methotrexate in an initial dose of $10 \mathrm{mg}$ per week which was increased to $15 \mathrm{mg}$ per week and a small dose of methylprednisolone.

In March 2016, the patient was re-hospitalized in the same department because of lower limb, shoulder girdle and knee pain, and calf edema. The patient reported night leg pain. Physical examination did not reveal symptoms of joint inflammation but extensive ulcers in both legs with purulent exudation were presented. Laboratory inflammatory markers were elevated, which is presented in Table I.

Ultrasonography of the joints and radiography findings matched osteoarthritis and did not support arthritis diagnosis at that moment. Therefore, methotrexate was discontinued and the patient was treated with antibiotics according to the wound culture. After the start of treatment outpatient care was recommended.

During the next hospitalization to the Department of Geriatrics in July 2016, the patient reported weakness, dyspnea, orthopnea, mouth and eye dryness, night pain of both lower limbs resulting in sleeplessness and excessive consumption of non-steroid anti-inflammatory analgesics. Due to general weakness and pain the patient spent most time in bed. The list of medications taken by the patient included bisoprolol, ramipril, indapamide, L-thyroxine, allopurinol, naproxen, tramadol, acetaminophen, omeprazole, ibandronic acid and cholecalciferol.

The examination revealed two extensive ulcerations of lower limbs covering around 2/3 of the right and half of the left calf circumference (Fig. 2A). The patient was hypotonic and hypervolemic. There was crepitation on both lung bases indicating heart failure. The peripheral arterial pulse was hardly palpable. Laboratory tests revealed anemia, elevated inflammatory markers, hyponatremia, and elevated $\mathrm{N}$-terminal pro-brain natriuretic peptide (NT-proBNP) (results summarized in Table I).

Table I. Results of laboratory tests

\begin{tabular}{|c|c|c|c|c|c|}
\hline Parameter & May 2014 & March 2016 & July 2016 & October 2016 & July 2018 \\
\hline RF & $20.0 \mathrm{IU} / \mathrm{ml}$ & $20.0 \mathrm{IU} / \mathrm{ml}$ & No data & No data & No data \\
\hline ACPA & $<7.00 \mathrm{U} / \mathrm{ml}$ & $<7.00 \mathrm{U} / \mathrm{ml}$ & No data & No data & No data \\
\hline ANA & $1: 80$ & $1: 640$ & No data & No data & No data \\
\hline CRP & $\begin{array}{c}58 \mathrm{mg} / \mathrm{l} \\
552.39 \mathrm{nmol} / \mathrm{l}\end{array}$ & $\begin{array}{c}33 \mathrm{mg} / \mathrm{l} \\
314.29 \mathrm{nmol} / \mathrm{l}\end{array}$ & $\begin{array}{c}45 \mathrm{mg} / \mathrm{l} \\
428.58 \mathrm{nmol} / \mathrm{l}\end{array}$ & $\begin{array}{c}19 \mathrm{mg} / \mathrm{l} \\
180.96 \mathrm{nmol} / \mathrm{l}\end{array}$ & $\begin{array}{c}11 \mathrm{mg} / \mathrm{l} \\
104.76 \mathrm{nmol} / \mathrm{l}\end{array}$ \\
\hline ESR & $56 \mathrm{~mm} / \mathrm{h}$ & $83 \mathrm{~mm} / \mathrm{h}$ & $95 \mathrm{~mm} / \mathrm{h}$ & $107 \mathrm{~mm} / \mathrm{h}$ & $56 \mathrm{~mm} / \mathrm{h}$ \\
\hline Creatinine & $\begin{array}{c}0.7 \mathrm{mg} / \mathrm{dl} \\
53.38 \mathrm{\mu mol} / \mathrm{l}\end{array}$ & $\begin{array}{c}2.0 \mathrm{mg} / \mathrm{dl} \\
152.50 \mu \mathrm{mol} / \mathrm{l}\end{array}$ & $\begin{array}{c}1.0 \mathrm{mg} / \mathrm{dl} \\
76.25 \mu \mathrm{mol} / \mathrm{l}\end{array}$ & $\begin{array}{c}0.9 \mathrm{mg} / \mathrm{dl} \\
68.63 \mu \mathrm{mol} / \mathrm{l}\end{array}$ & $\begin{array}{c}1.10 \mathrm{mg} / \mathrm{dl} \\
83.88 \mu \mathrm{mol} / \mathrm{l}\end{array}$ \\
\hline NT-proBNP & No data & No data & $\begin{array}{c}1100 \mathrm{pg} / \mathrm{ml} \\
130.06 \mathrm{pmol} / \mathrm{l}\end{array}$ & $\begin{array}{c}936.8 \mathrm{pg} / \mathrm{ml} \\
110.77 \mathrm{pmol} / \mathrm{l}\end{array}$ & $\begin{array}{l}783.5 \mathrm{pg} / \mathrm{ml} \\
92.64 \mathrm{pmol} / \mathrm{l}\end{array}$ \\
\hline
\end{tabular}

RF-rheumatoid factor, ACPA - anti-cyclic citrullinated peptide antibodies, ANA - antinuclear antibodies, CRP - C-reactive protein, ESR - erythrocyte sedimentation rate, NT-proBNP - N-terminal pro-brain natriuretic peptide. 
Klebsiella pneumoniae and Serratia marcescens strains were cultured from the ulcers. Chronic heart failure exacerbation (NYHA III/IV), chronic kidney disease exacerbation, anemia of chronic disease and mixed-etiology leg ulcers were diagnosed.

Wounds were debrided, topical and systemic (empirical and targeted) antibiotics were used, analgesics, diuretics were administered and dressings were applied. Due to a significant reduction in hemoglobin concentration and to improve healing conditions it was necessary to transfuse the red blood cell concentrate. No evident positive effect was achieved in treatment of legs ulcers, which remained deep, inflamed, with purulent discharge. Resting lower limb pain persisted. The Doppler ultrasound revealed occlusion of the right femoral artery. The patient was referred for surgery in which femoral-popliteal bypass surgery was performed. A significant reduction in leg edema was observed. Pain complaints resolved almost completely.
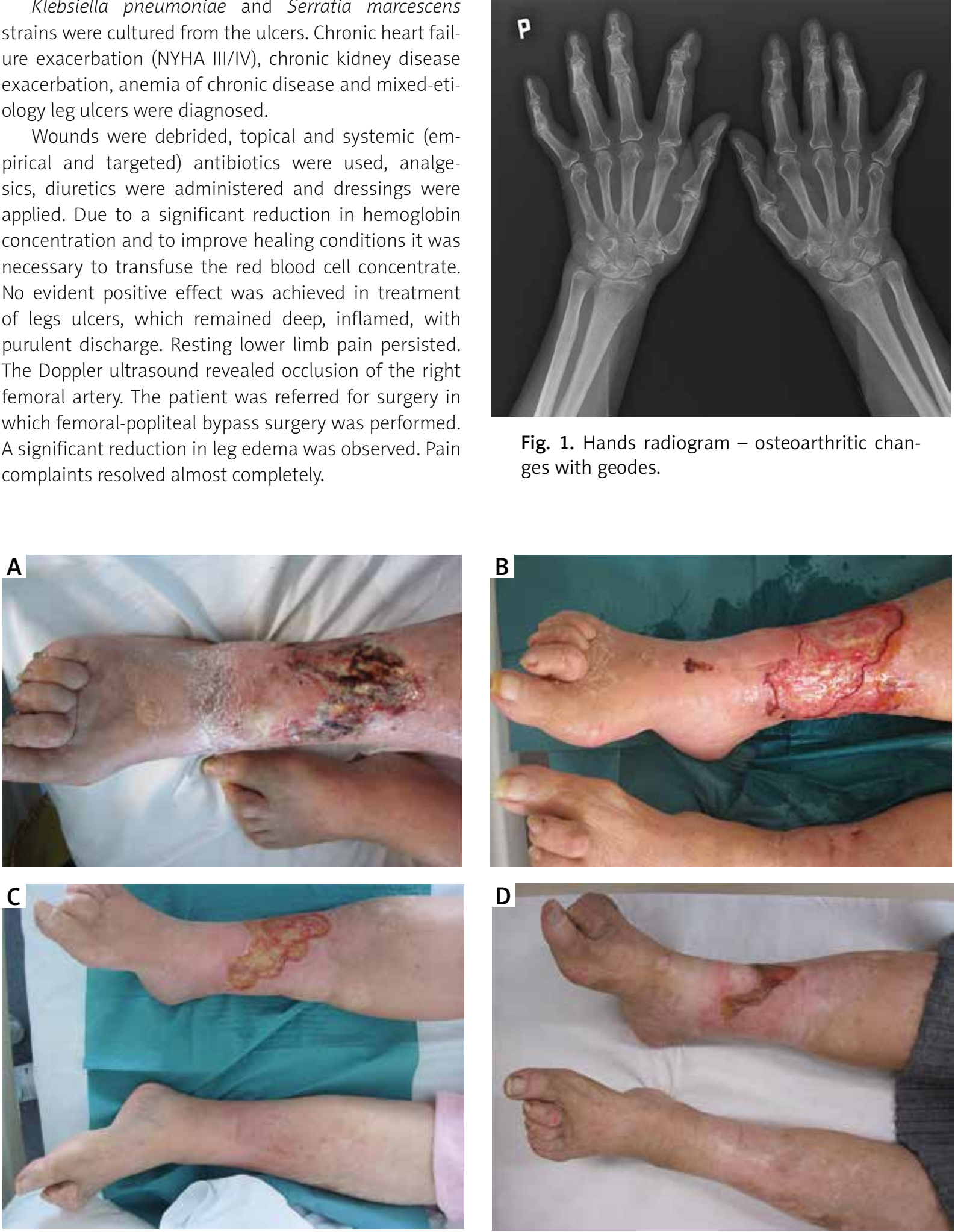

Fig. 1. Hands radiogram - osteoarthritic changes with geodes.

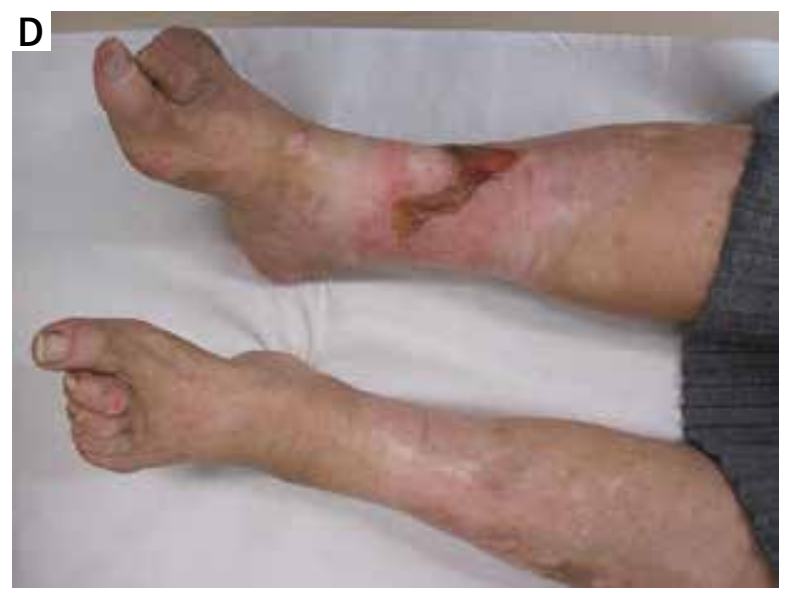

Fig. 2. Legs ulcerations - healing progress. (A) 13.07.2016, (B) 13.10.2016, (C) 27.01.2017, (D) 19.10.2017. 
In October 2016, about 3 months after the bypass surgery, the patient was admitted to a regional hospital with critical ischemia of the right lower limb suspected, with accompanying inflammation. Leg amputation was considered, but the patient did not agree to such radical treatment. Therefore the patient was transferred to the Department of Geriatrics for conservative treatment. On admission physical examination revealed extensive deep ulceration of $2 / 3$ of the right calf circumference and two ulcerations on the outer surface of the left calf with dimensions comparable to those during the previous discharge (Fig. 2B), palpable right inguinal lymph nodes, which were confirmed in ultrasound, and large subcutaneous edema, especially of the right limb.

Based on a repeated wound smear culture, targeted ceftazidime was administered intravenously for 11 days. A very slow healing process was noted, and microvascular disorders were taken into consideration as a decisive factor for the impaired healing. Actual cardiology treatment was modified - beta-blocker treatment was replaced with a non-dihydropyridine calcium channe blocker because of a suspected mechanism of peripheral microvascular deterioration by beta-receptor inhibition. Also a vasodilatory drug (bencyclane) was added.

In the next two weeks a significant reduction in the depth and dimensions of ulcers was observed. Control wound smear cultures were negative. The patient learned to walk with crutches and was discharged to outpatient care. The patient was informed about proper, intensified wound hygiene and change of dressings.

In the next few weeks, the healing of the left leg ulcer was complete and the right leg ulcer reduced to superficial and $1 / 3$ of the previous size in a follow-up after several months (Figs. 2C and 2D). At the follow-up in July 2018, both legs were cicatrized, the patient was self-reliant in basic daily activities, walking on crutches, painless.

\section{Discussion}

Contemporary medicine offers a broad choice of therapies in leg ulcer healing, varying from conservative treatment with vasodilatory drugs, more and more sophisticated wound dressings, through more aggressive therapy for underlying autoimmune using disease-modifying antirheumatic drugs and biological agents such as TNF- $\alpha$ inhibitors and rituximab as treatment for RA-associated leg ulcers [6], to vascular surgery and split skin grafting [5].

This case report is intended to underline the role of differential diagnosis in the decision-making process of selecting the appropriate therapy in lower limb ulcers. The proper diagnosis of all factors in ulcer pathogenesis leads to the selection of a suitable therapy and successful healing. Studies showed that glucocorticoids may predispose to skin fragility and thus serve as a risk factor for ulceration in patients with RA and potential other autoimmune diseases as Sjögren's syndrome. Sometimes it can be difficult to differentiate whether the cause of ulceration is the effect of the disease itself or an adverse effect of the treatment applied for this disease.

In Sjögren syndrome, leg ulcers may occur as a consequence of leukocytoclastic vasculitis [7]. In such cases, skin biopsy should be performed and other systemic vasculitides should be considered in the differential diagnosis. Laboratory testing for component of complement, anti-neutrophil antibodies and cryoglobulins is indicated. Signs of inflammatory vasculitis ulcers are very similar or the same as in more often ischemic or venous ulcers.

In this case, the diagnosis of Sjögren syndrome remains controversial. Unfortunately, there is no available objective medical documentation prior to 2014, and all data come from the anamnesis. The patient refused a salivary gland biopsy in the past. Moreover, starting from 2014, the patient did not fulfill the criteria of primary Sjögren syndrome at any stage of the therapy. Rheumatoid factor was negative, ANA titer was initially negative, only in 2016 they were $1: 640$ but without specificity. Anti-Ro/SSA, La/SSB and other ENA antibodies were not detected by dot-blot method in particular [8]. Maślińska et al. [9] describe a positive correlation between ANA titer, anti-Ro, anti-La antibodies and erythrocyte sedimentation rate (ESR) in patients with primary Sjögren syndrome, as important factors in the inflammatory process. Elevated C-reactive protein (CRP) and ESR in the present case were not associated with an autoimmune disease, but with a bacterial wound infection. Thus, we did not perform a skin biopsy and other immune tests. It is worth remembering that a geriatric patient can be afebrile even in a serious infection, and the only signs and symptoms could be discrete changes of mental state, weakness, falls, etc.

We believe that the reported mouth and eye dryness along with the positive Schirmer's test were consequences of polypragmasy and the aging process. Smidt et al. [10] found in their cohort study that oral and ocular dryness in older people is correlated with the number of medications used and the number of comorbidities. A diagnosis of more than four diseases was related to $19.7 \%$ prevalence of oral and $16.4 \%$ prevalence of ocular dryness; the use of more than 6 medications was related to $19.6 \%$ prevalence of both. High prevalence and odds ratios for mouth dryness was associated with metabolic diseases (31\%), intake of thyroid hormones (27\%), beta-blockers (11.8\%), paracetamol, and opioids 
(33.3\%), proton pump inhibitors (15.9\%), bisphosphonates (25\%), diuretics (12.8\%) and ACE inhibitors (12\%). The patient described was treated with a majority of medications mentioned above.

Leg ulcers emerged about six months after the treatment with methotrexate had started. This immunosuppressive agent changes the immune response to microbes, which in our opinion was crucial for this patient. Even a small injury to the skin exposed to glucocorticosteroids for many years could become easily infected. Unfavorable healing conditions, such as atherosclerotic ischemia, impaired venous flow and immobilization caused by pain promote deep and infected ulcerations. Unfortunately, in 2014, the clinical and radiological image was too unclear to definitely exclude autoimmune arthritis, and the patient was lost from observation for the next two years.

All these factors resulted in a "net disease" with ulcers of complex etiology as a main symptom. Although the patient received multidisciplinary treatment, a simple change of pharmacotherapy could rupture the net.

Many studies evaluate positive effects of different approaches, but there are very few publications addressing adverse effects of joint therapies which may have detrimental effect on healing. One of our considerations is deterioration of microcirculation caused by the use of beta-blockers. While there is no doubt as to the beneficial influence of this pharmacological class on the circulatory system, we want to point out some specific situations, when these drugs could be harmful. Especially the population of elderly patients, with the burden of atherosclerosis involving small blood vessels, could be prone to deterioration of blood flow due to the blockade of peripheral $\beta$-1 receptors, even despite the use of a cardioselective drug (nebivolol) with a vasodilatory component, as we observed in the case described herein.

\section{Conclusions}

It seems that despite the lack of clear recommendations concerning this issue [11-13], replacing the betablocker with a non-dihydropyridine calcium channel blocker could be critical for healing leg ulcers in some groups of elderly patients.

\section{Acknowledgments}

The authors would like to thank Professor Iwona Sudoł-Szopinska, Head of the Radiology Department in the National Institute of Geriatrics, Rheumatology and Rehabilitation for providing them with access to the radiology imaging, and would like to address words of par- ticular gratitude to Doctor Agnieszka Skoczylas for her valuable comments concerning their paper.

The authors declare no conflicts of interest.

\section{References}

1. Mekkes JR, Loots MA, Van Der Wal AC, Bos JD. Causes, investigation and treatment of leg ulceration. Br J Dermatol 2003; 148: 388-401

2. Tendera M, Aboyans V, Barterlink ML, et al. and ESC Committee for Practice Guidelines. ESC Guidelines on the diagnosis and treatment of peripheral artery diseases: Document covering atherosclerotic disease of extracranial carotid and vertebral, mesenteric, renal, upper and lower extremity arteries: the Task Force on the Diagnosis and Treatment of Peripheral Artery Diseases of the European Society of Cardiology (ESC). Eur Heart J 2011; 32: 2851-2906.

3. Shanmugam VK, Angra D, Rahim H, McNish S. Vasculitic and autoimmune wounds. J Vasc Surg Venous Lymphat Disord 2017; 5: 280-292.

4. Moffatt CJ, Franks PJ, Doherty DC, et al. Prevalence of leg ulceration in a London population. QIM 2004; 97: 431-471.

5. Hafne J, Schneider E, Burg G, Cassina PC. Management of leg ulcers in patients with rheumatoid arthritis or systemic sclerosis: The importance of concomitant arterial and venous disease. J Vasc Surg 2000; 32: 322-329.

6. Shanmugam VK. Vasculitic Diseases and Prothrombotic States Contributing to Delayed Healing in Chronic Wounds. Curr Dermatol Rep 2016; 5: 270-277.

7. Chapnick SL, Merkel PA. Skin ulcers in a patient with Sjogren's Syndrome. Arthritis Care Res (Hoboken) 2010; 62: 1040-1046.

8. Aletaha D, Neogi T, Silman AJ, et al. 2010 Rheumatoid arthritis classification criteria: an American College of Rheumatology/ European League Against Rheumatism collaborative initiative. Arthritis Rheum 2010; 62: 2569-2581.

9. Maślińska M, Kontny E, Kwiatkowska B. The relationship between the presence of autoantibodies, indicators of local and systemic inflammation, the serum concentration of B-cell activating factor (BAFF) and the intensity of salivary gland infiltration in patients with primary Sjögren's syndrome - a preliminary study. Reumatologia 2015; 53: 321-327.

10. Smidt D, Torpet LA, Nauntofte B. Associations between oral and ocular dryness, labial and whole salivary flow rates, systemic diseases and medications in a sample of older people. Community Debt Oral Epidemiol 2011; 39: 276-288.

11. Frołow M, Leśniak W, Masłowski L. Przewlekłe niedokrwienie kończyn dolnych. In: Interna Szczeklika, Medycyna Praktyczna, Kraków 2018: 496-503.

12. Paravastu SC, Mendonca DA, Da Silva A. Beta blockers for peripheral arterial disease. Cochrane Database Syst Rev 2013: CD005508.

13. Csiki Z, Garai I, Shemirani AH, et al. The effect of metoprolol alone and combined metoprolol-felodipin on the digital microcirculation of patients with primary Raynaud's syndrome. Microvasc Res 2011; 82: 84-87. 